Supplementary Information for:

\title{
Graphene Layer Morphology as an Indicator of the Metals Alloy Formation at the Interface
}

\author{
Elena Voloshina, ${ }^{*},+\ddagger$ Beate Paulus ${ }^{\ddagger}{ }^{\ddagger}$ and Yuriy Dedkov ${ }^{*} \dagger$ \\ †Department of Physics, Shanghai University, 200444 Shanghai, China \\ $\ddagger$ Institut für Chemie und Biochemie, Freie Universität Berlin, Arnimallee 22, 14195 \\ Berlin, Germany \\ E-mail: voloshina@shu.edu.cn; dedkov@shu.edu.cn
}




\section{List of figures and tables:}

Fig. S1. STM images acquired after deposition of $\mathrm{Mn}$ on $\operatorname{gr} / \mathrm{Ru}(0001)$ and $\operatorname{gr} / \operatorname{Ir}(111)$. $\mathrm{Mn}+\mathrm{gr} / \mathrm{Ru}(0001): \quad(\mathrm{a}) 120 \times 120 \mathrm{~nm}^{2}, U_{T}=+300 \mathrm{mV}, I_{T}=1 \mathrm{nA}, \quad\left(\right.$ b) $17 \times 17 \mathrm{~nm}^{2}$, $U_{T}=+100 \mathrm{mV}, I_{T}=15 \mathrm{nA} ; \mathrm{Mn} / \mathrm{gr}$-islands/Ir(111): (c) $180 \times 180 \mathrm{~nm}^{2}, U_{T}=+300 \mathrm{mV}$, $I_{T}=1 \mathrm{nA},(\mathrm{d}) 50 \times 29 \mathrm{~nm}^{2}, U_{T}=+300 \mathrm{mV}, I_{T}=1 \mathrm{nA} ; \mathrm{Mn} / \mathrm{gr} / \operatorname{Ir}(111):(\mathrm{e}) 120 \times 120 \mathrm{~nm}^{2}$, $U_{T}=+700 \mathrm{mV}, I_{T}=0.5 \mathrm{nA},\left(\right.$ f) $30 \times 30 \mathrm{~nm}^{2}, U_{T}=+500 \mathrm{mV}, I_{T}=0.5 \mathrm{nA},(\mathrm{g}) 12 \times 8 \mathrm{~nm}^{2}$, $U_{T}=+50 \mathrm{mV}, I_{T}=15 \mathrm{nA}$. In panel (c), Mn/gr/Ir(111) and $\mathrm{Mn} / \operatorname{Ir}(111)$ areas are marked with $\mathrm{A}$ and $\mathrm{B}$, respectively.

Fig. S2. STM images acquired after intercalation of $\mathrm{Mn}$ in $\mathrm{gr} / \mathrm{Ru}(0001)$ and $\mathrm{gr} / \mathrm{Ir}(111)$. gr/Mn-Ru(0001): (a) $60 \times 60 \mathrm{~nm}^{2}, U_{T}=+100 \mathrm{mV}, I_{T}=1 \mathrm{nA}$, (b) $8 \times 8 \mathrm{~nm}^{2}, U_{T}=+100 \mathrm{mV}$, $I_{T}=1 \mathrm{nA} ; \mathrm{gr} / \mathrm{Mn}-\operatorname{Ir}(111):(\mathrm{c}) 120 \times 50 \mathrm{~nm}^{2}, U_{T}=+100 \mathrm{mV}, I_{T}=2 \mathrm{nA}$, (d) $26 \times 26 \mathrm{~nm}^{2}$, $U_{T}=-100 \mathrm{mV}, I_{T}=15 \mathrm{nA}$, (e) $6 \times 6 \mathrm{~nm}^{2}, U_{T}=-100 \mathrm{mV}, I_{T}=5 \mathrm{nA}$. White and black frames in (c) and (d) mark the zoomed areas presented in (d) and (e), respectively.

Tab. S3. Overview of the systems and structures considered in the present work and used in the DFT calculations: top view of the system (blue rhombus mark the unit cell); calculated STM images for $U_{T}= \pm 300 \mathrm{mV}$ (marked in the figure); composition of the graphene-metal slab used in the DFT calculations; total energy of the system and the respective change of the total energy with respect to the one for the gr/Mn-M/M slab; averaged and minimal distances between graphene and the top metal layer in the considered system and the respective corrugation a graphene monolayer.

Fig. S4. Theoretically simulated STM image of gr/Ir/MnIr/Ir(111). The integration of the electronic states around $E_{F}$ was performed for the energy range corresponding to the experimental bias voltage of $U_{T}=-300 \mathrm{mV}$ and the obtained images correspond to the experimental tunneling current of $I_{T} \approx 1.2 \mathrm{nA}$. Inset shows the respective FFT image. 

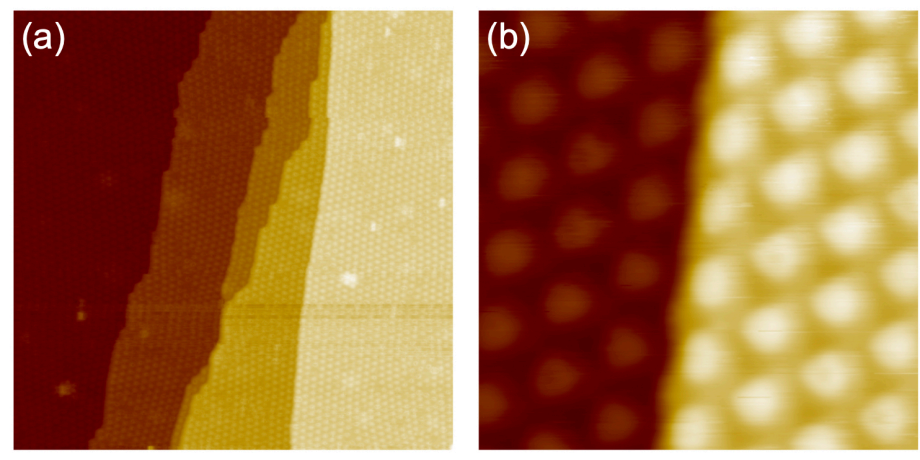

\section{Mn/gr-islands/lr(111)}
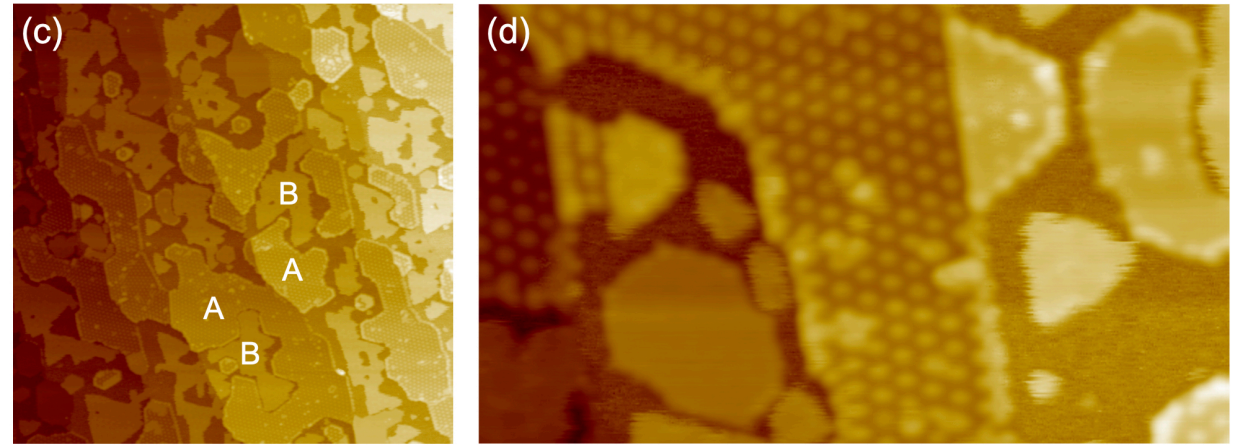

\section{$\mathrm{Mn} / \mathrm{gr} / \operatorname{lr}(111)$}
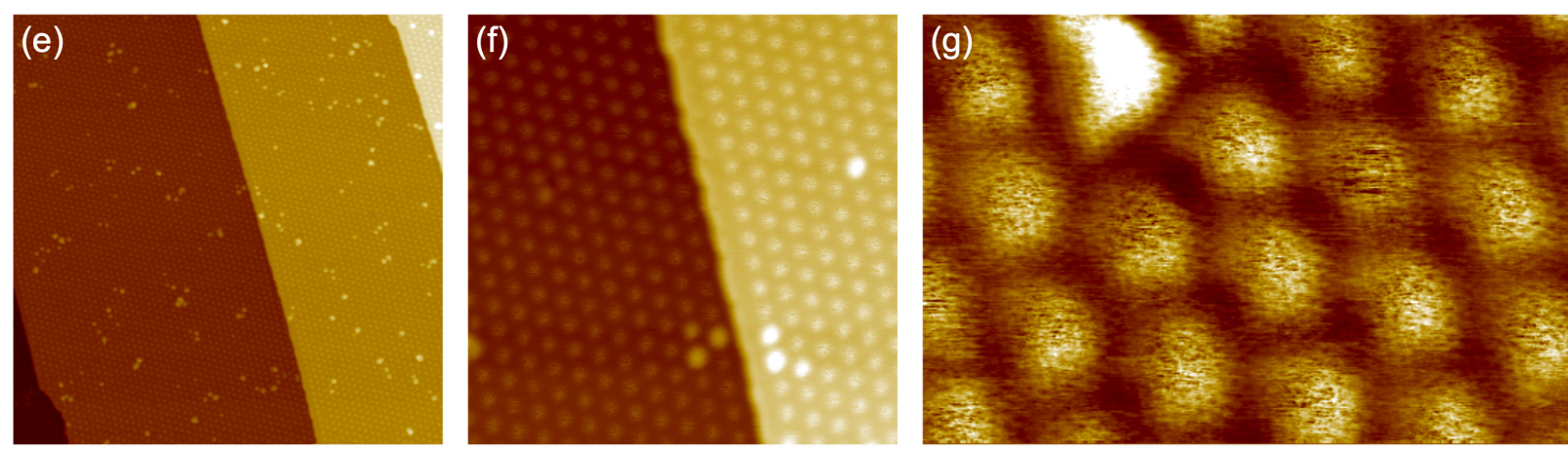

Fig. S1. STM images acquired after deposition of $\mathrm{Mn}$ on $\operatorname{gr} / \mathrm{Ru}(0001)$ and $\operatorname{gr} / \operatorname{Ir}(111)$. $\mathrm{Mn}+\mathrm{gr} / \mathrm{Ru}(0001): \quad$ (a) $120 \times 120 \mathrm{~nm}^{2}, U_{T}=+300 \mathrm{mV}, I_{T}=1 \mathrm{nA},\left(\right.$ b) $17 \times 17 \mathrm{~nm}^{2}$, $U_{T}=+100 \mathrm{mV}, I_{T}=15 \mathrm{nA} ; \mathrm{Mn} / \mathrm{gr}-i s l a n d s / \operatorname{Ir}(111):(\mathrm{c}) 180 \times 180 \mathrm{~nm}^{2}, U_{T}=+300 \mathrm{mV}$, $I_{T}=1 \mathrm{nA},(\mathrm{d}) 50 \times 29 \mathrm{~nm}^{2}, U_{T}=+300 \mathrm{mV}, I_{T}=1 \mathrm{nA} ; \mathrm{Mn} / \mathrm{gr} / \operatorname{Ir}(111):(\mathrm{e}) 120 \times 120 \mathrm{~nm}^{2}$, $U_{T}=+700 \mathrm{mV}, I_{T}=0.5 \mathrm{nA}$, (f) $30 \times 30 \mathrm{~nm}^{2}, U_{T}=+500 \mathrm{mV}, I_{T}=0.5 \mathrm{nA},(\mathrm{g}) 12 \times 8 \mathrm{~nm}^{2}$, $U_{T}=+50 \mathrm{mV}, I_{T}=15 \mathrm{nA}$. In panel (c), Mn/gr/Ir(111) and $\mathrm{Mn} / \operatorname{Ir}(111)$ areas are marked with $\mathrm{A}$ and $\mathrm{B}$, respectively. 
gr/Mn-Ru(0001)
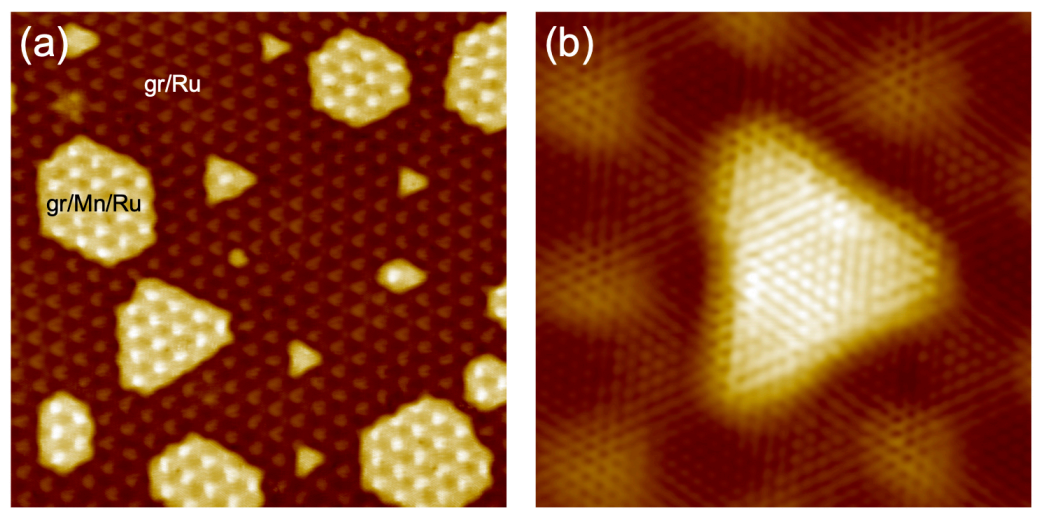

gr/Mn-Ir(111)
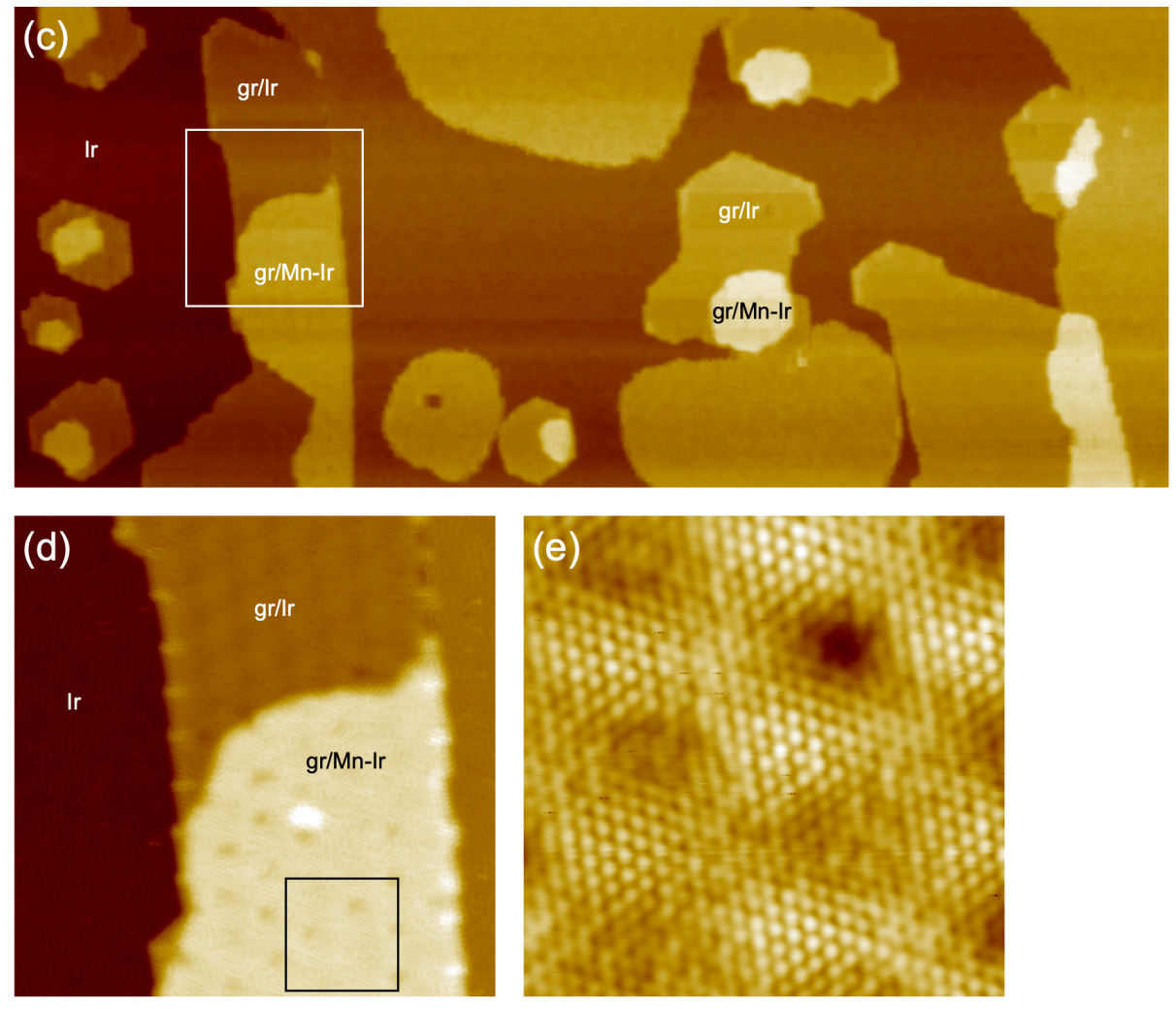

Fig. S2. STM images acquired after intercalation of $\mathrm{Mn}$ in $\mathrm{gr} / \mathrm{Ru}(0001)$ and $\mathrm{gr} / \mathrm{Ir}(111)$. gr/Mn-Ru(0001): (a) $60 \times 60 \mathrm{~nm}^{2}, U_{T}=+100 \mathrm{mV}, I_{T}=1 \mathrm{nA}$, (b) $8 \times 8 \mathrm{~nm}^{2}, U_{T}=+100 \mathrm{mV}$, $I_{T}=1 \mathrm{nA} ; \mathrm{gr} / \mathrm{Mn}-\operatorname{Ir}(111):\left(\right.$ c) $120 \times 50 \mathrm{~nm}^{2}, U_{T}=+100 \mathrm{mV}, I_{T}=2 \mathrm{nA}$, (d) $26 \times 26 \mathrm{~nm}^{2}$, $U_{T}=-100 \mathrm{mV}, I_{T}=15 \mathrm{nA}$, (e) $6 \times 6 \mathrm{~nm}^{2}, U_{T}=-100 \mathrm{mV}, I_{T}=5 \mathrm{nA}$. White and black frames in (c) and (d) mark the zoomed areas presented in (d) and (e), respectively. 
Tab. S3. Overview of the systems and structures considered in the present work and used in the DFT calculations: top view of the system (blue rhombus mark the unit cell); calculated STM images for $U_{T}= \pm 300 \mathrm{mV}$ (marked in the figure); composition of the graphene-metal slab used in the DFT calculations; total energy of the system and the respective change of the total energy with respect to the one for the gr/Mn-M/M slab; averaged and minimal distances between graphene and the top metal layer in the considered system and the respective corrugation a graphene monolayer.

\begin{tabular}{|c|c|c|c|c|c|}
\hline $\begin{array}{c}\text { Structure } \\
\text { (top view })\end{array}$ & $\begin{array}{c}\text { STM } \\
(-/+300 \mathrm{mV})\end{array}$ & $\begin{array}{c}\text { Composition } \\
(\text { atoms })\end{array}$ & $\begin{array}{c}\text { Energy } \\
(\mathrm{eV})\end{array}$ & $\begin{array}{c}\text { Distances } \\
(\AA)\end{array}$ \\
\hline \multicolumn{2}{|c|}{ graphene/Ru(0001) } & $\mathrm{C}: \quad 338$ & total: -9965.985 & avg.: & 2.380 \\
\hline \\
\hline
\end{tabular}

graphene/Mn/Ru(0001)

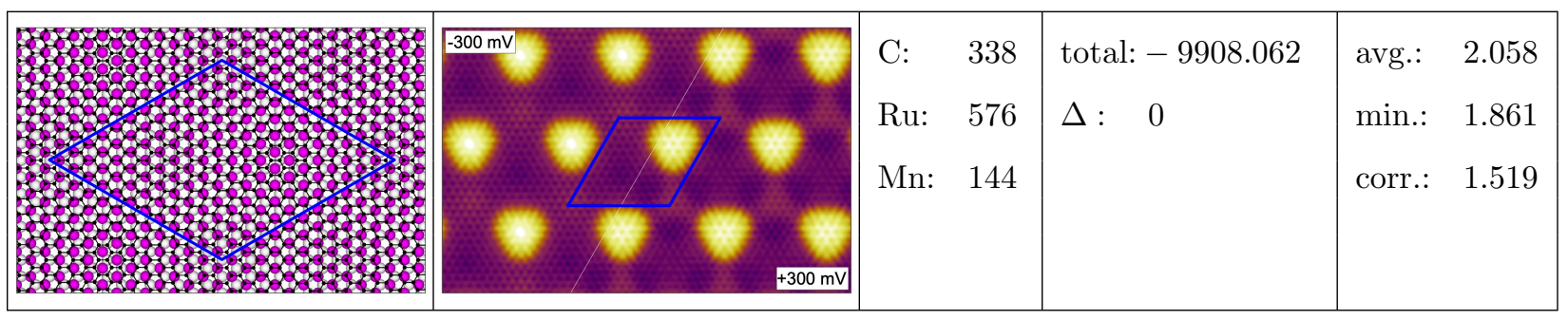

graphene/Ru/Mn/Ru(0001)

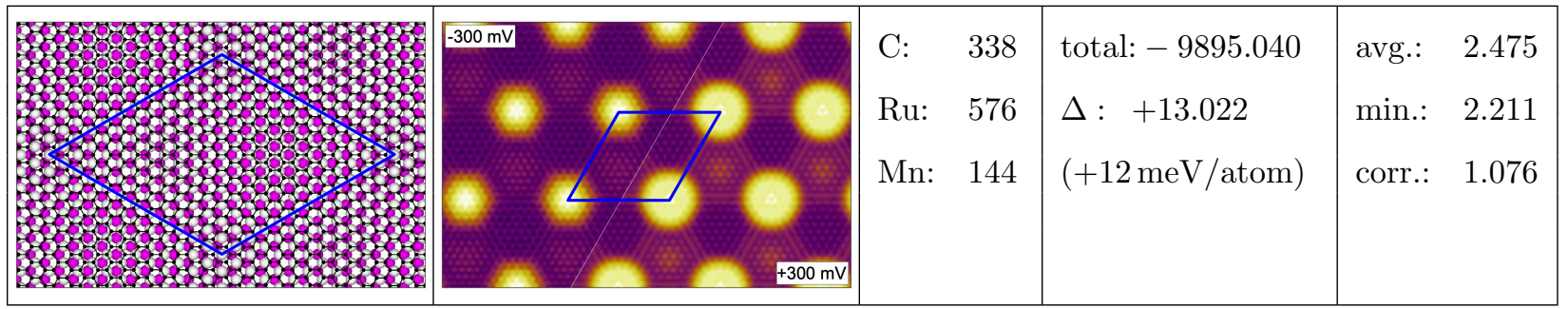

graphene/Ir(111)

\begin{tabular}{|c|c|c|c|c|}
\hline 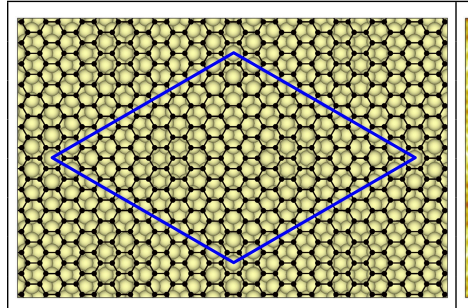 & 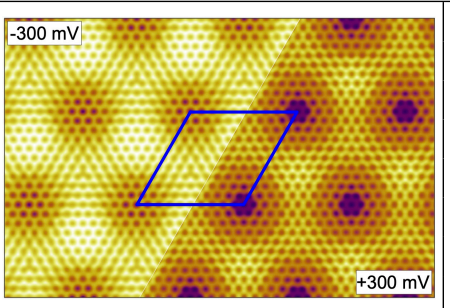 & $\begin{array}{ll}\text { C: } & 200 \\
\text { Ir: } & 405\end{array}$ & total: -5434.135 & $\begin{array}{ll}\text { avg.: } & 3.397 \\
\text { min.: } & 3.289 \\
& \\
\text { corr.: } & 0.358\end{array}$ \\
\hline
\end{tabular}


Tab. S3 - continued from previous page

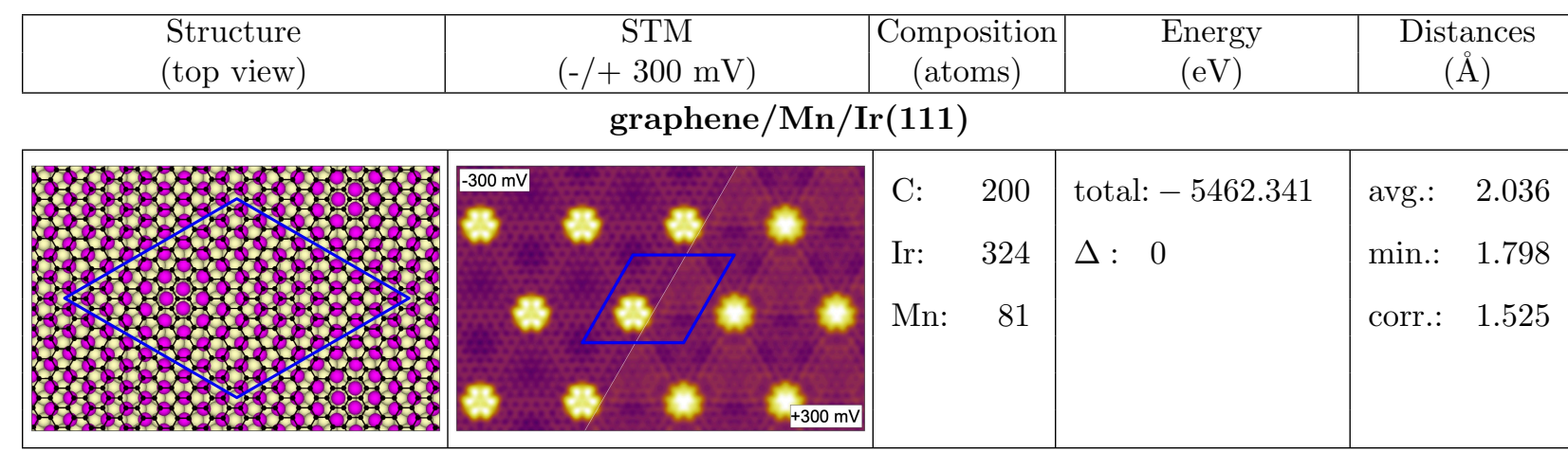

graphene/Ir/Mn/Ir(111)

\begin{tabular}{|c|c|c|c|c|c|}
\hline 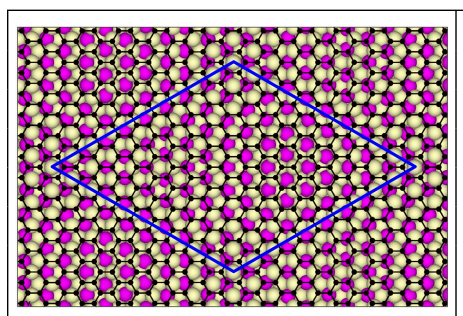 & 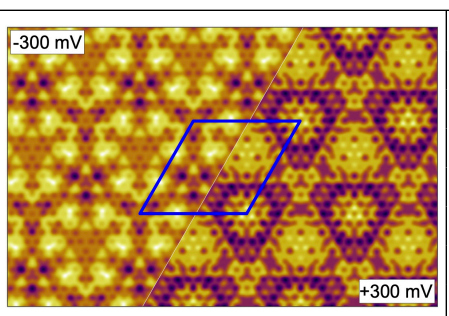 & $\begin{array}{l}\text { C: } \\
\text { Ir: } \\
\text { Mn: }\end{array}$ & $\begin{array}{r}200 \\
324 \\
81\end{array}$ & $\begin{array}{l}\text { total: }-5475.710 \\
\Delta: \quad-13.369 \\
(-22 \mathrm{meV} / \text { atom })\end{array}$ & $\begin{array}{ll}\text { avg.: } & 3.375 \\
\text { min.: } & 3.283 \\
\text { corr.: } & 0.278\end{array}$ \\
\hline
\end{tabular}

graphene/IrMn/Ir(111) (ordered)

\begin{tabular}{|c|c|c|c|c|c|}
\hline 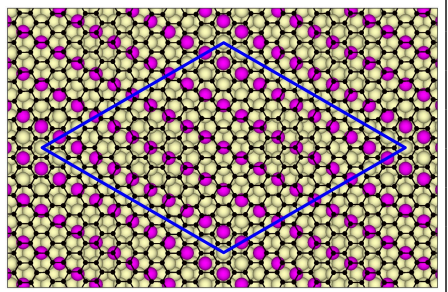 & (2) & $\begin{array}{ll}\text { C: } & 242 \\
\text { Ir: } & 450 \\
\text { Mn: } & 50\end{array}$ & $\begin{array}{l}\text { total: }-6677.367 \\
\Delta: \quad 0\end{array}$ & $\begin{array}{l}\operatorname{avg} .: \\
\min .: \\
\text { corr.: }\end{array}$ & $\begin{array}{l}2.730 \\
1.982 \\
1.897\end{array}$ \\
\hline
\end{tabular}

graphene/Ir/IrMn/Ir(111) (ordered)
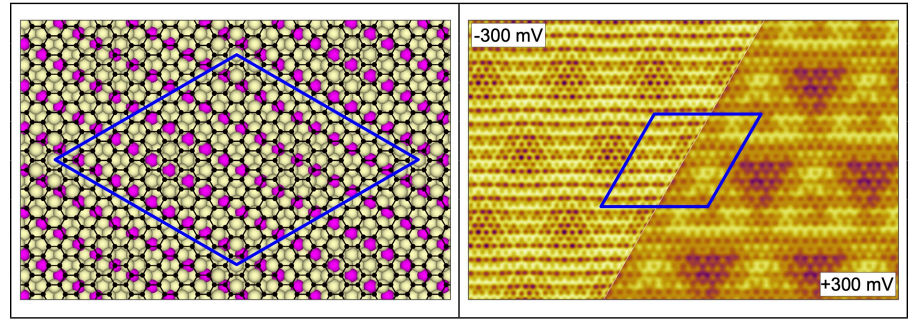

\begin{tabular}{lr|l|ll|} 
C: & 242 & total: -6705.638 & avg.: & 3.349 \\
Ir: & 450 & $\Delta:-28.272$ & min.: & 3.224 \\
Mn: & 50 & $(-38.1 \mathrm{meV} /$ atom $)$ & corr.: & 0.429 \\
& & & \\
\hline
\end{tabular}


Tab. S3 - continued from previous page

\begin{tabular}{|c|c|c|c|c|}
\hline $\begin{array}{l}\text { Structure } \\
\text { (top view) }\end{array}$ & $\begin{array}{c}\text { STM } \\
(-/+300 \mathrm{mV})\end{array}$ & $\begin{array}{c}\text { Composition } \\
\text { (atoms) }\end{array}$ & $\begin{array}{c}\text { Energy } \\
(\mathrm{eV})\end{array}$ & $\begin{array}{c}\text { Distances } \\
(\AA)\end{array}$ \\
\hline \multicolumn{5}{|c|}{ graphene/IrMn/Ir(111) (disordered) } \\
\hline 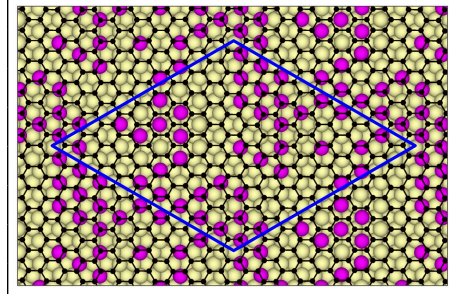 & & $\begin{array}{lr}\text { C: } & 200 \\
\text { Ir: } & 364 \\
\text { Mn: } & 41\end{array}$ & $\begin{array}{l}\text { total: }-5442.385 \\
\Delta: 0\end{array}$ & $\begin{array}{ll}\text { avg.: } & 2.622 \\
\min .: & 1.812 \\
\text { corr.: } & 1.941\end{array}$ \\
\hline
\end{tabular}

graphene/Ir/IrMn/Ir(111) (disordered)

\begin{tabular}{|c|c|c|c|c|c|}
\hline 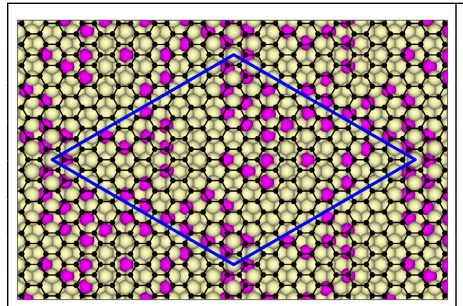 & 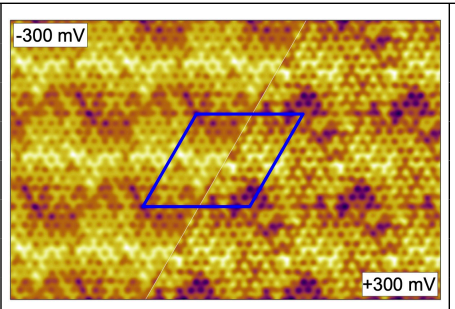 & $\begin{array}{ll}\text { C: } & 2 \\
\text { Ir: } & 3 \\
\text { Mn: } & \end{array}$ & $\begin{array}{l}200 \\
364 \\
41\end{array}$ & $\begin{array}{l}\text { total: }-5454.273 \\
\Delta:-11.888 \\
(-19.6 \mathrm{meV} / \text { atom })\end{array}$ & $\begin{array}{ll}\text { avg.: } & 3.371 \\
\text { min.: } & 3.256 \\
\text { corr.: } & 0.277\end{array}$ \\
\hline
\end{tabular}




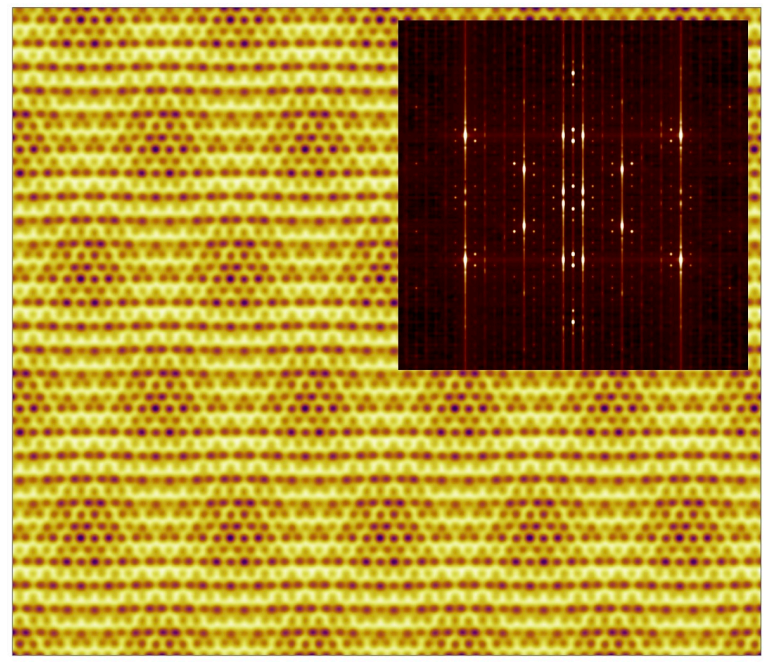

Fig. S4. Theoretically simulated STM image of $\mathrm{gr} / \mathrm{Ir} / \mathrm{MnIr} / \operatorname{Ir}(111)$. The integration of the electronic states around $E_{F}$ was performed for the energy range corresponding to the experimental bias voltage of $U_{T}=-300 \mathrm{mV}$ and the obtained images correspond to the experimental tunneling current of $I_{T} \approx 1.2 \mathrm{nA}$. Inset shows the respective FFT image. 